\title{
Desempenho reprodutivo de nulíparas suínas artificialmente inseminadas no segundo, terceiro ou quarto estro pós-alojamento*
}

\author{
Reproductive performance of nuliparous sows first mated at second, \\ third or fourth oestrus after housing
}

\author{
Fabiane Mendonça Ferreira ${ }^{1}$, Guilherme Borchardt Neto ${ }^{1} \&$ Débora Elfrida Schröeder ${ }^{2}$ \\ RESUMO
}

O momento da primeira cobertura ou inseminação artificial (IA) da nulípara pode influenciar a eficiência produtiva do plantel suíno. O objetivo deste trabalho foi investigar a influência do estro da IA sobre a performance reprodutiva de fêmeas suínas em duas parições consecutivas. Trezentas e dezesseis fêmeas híbridas foram distribuidas em três tratamentos: tratamento 1 (T1), fêmeas inseminadas no segundo estro pós-alojamento (n=103); tratamento 2 (T2), fêmeas inseminadas no terceiro estro pós-alojamento $(n=104)$ e tratamento 3 (T3), fêmeas inseminadas no quarto estro pós-alojamento ( $n=109)$. As taxas de retorno ao estro e as taxas de parto foram comparadas pelo teste Exato de Fischer. As taxas de descarte no período entre o $1^{\mathrm{o}}$ parto e a $2^{\mathrm{a}}$ IA e o porcentual de fêmeas que completaram o segundo parto foram comparados pelo teste do Qui-quadrado. A análise de variância foi aplicada para avaliar o número de leitões nascidos e comparado pelo teste $t$ de Student. Não houve diferença quanto à taxa de retorno ao estro, taxa de parto e número de leitões nascidos no primeiro parto das fêmeas dos três tratamentos ( $\mathrm{p}>0,05)$. A taxa de descarte no período entre o $1^{\circ}$ parto e a próxima IA foi de $22,62 \%$, 30,86 \% e 20,23 \% para as fêmeas do T1, T2 e T3 (P>0,10), respectivamente. No segundo parto, as fêmeas do T1 apresentaram maior taxa de retorno ao estro $(\mathrm{P}<0,05)$, menor taxa de parto $(\mathrm{P}<0,05)$ e menor número de leitões nascidos $(\mathrm{P}=0,06)$, comparativamente ao T2 e T3. Os resultados mostram não haver diferença entre os tratamentos $(\mathrm{p}>0,05)$ no número de fêmeas que completaram dois partos. A performance inferior das fêmeas do T1 em relação à taxa de parto na segunda gestação comparativamente ao T2 e T3 foi parcialmente compensada pelo desempenho numericamente superior na primeira gestação.

Descritores: primíparas, estro de inseminação, taxa de parto, tamanho de leitegada, retorno ao estro.

\begin{abstract}
The time of the first mating or artificial insemination (AI) of the gilt can influence the productive efficiency of the swine herd. The present study was performed to evaluate the influence of oestrus at the first AI on reproductive performance of sows in two consecutive parities. Three hundred and sixteen hibrid sows were distributed in three treatments: treatment 1 (T1), sows inseminated at second oestrus after housing $(\mathrm{n}=103)$; treatment 2 (T2), sows inseminated at third oestrus after housing $(n=104)$, and treatment 3 (T3), sows inseminated at fourth oestrus after housing ( $n=109)$. Return to oestrus rate and parity rate were compared by Fischer Test. Culling rate between parity one and the second AI, and the percentage of females that completed the second parity were compared by Chi-Square test. Analysis of variance was applied to evaluate the number of piglets born, and compared by T-Student Test. There was no difference between treatments in relation to return to oestrus rate, parity rate, and piglets born in first parity ( $p>0.05)$. Culling rate between parity one and next AI were 22.62\%,30.86\%, and 20.23\% to females from T1, T2, and T3 (p>0.10), respectively. In parity two, females from $\mathrm{T} 1$ showed greater return to oestrus rate $(\mathrm{p}<0.05)$, lower parity rate $(\mathrm{p}<0.05)$, and lower number of piglets born $(\mathrm{p}<0.06)$, comparatively to T2 and T3. Results show no difference between treatments on number of sows completing two parities. The inferior performance of T1 sows in terms of party rate in parity two comparatively to T2 and T3 females was partially compensated by numerical superior performance in first gestation.
\end{abstract}

Key words: primiparous sow, oestrus of insemination, parity rate, litter size, return to oestrus. 


\section{INTRODUÇÃO}

A eficiência reprodutiva das fêmeas é determinada em grande parte pela precocidade sexual e possibilidade de introdução igualmente precoce no plantel de reprodução. De maneira geral, leitoas de reposição são incorporadas à criação antes de manifestarem a puberdade. Até o momento da cobertura ou inseminação artificial (IA), são considerados animais improdutivos dentro do sistema criatório, podendo contribuir para aumentar substancialmente o número de dias não produtivos do plantel.

De um modo geral, a norma de manejo que orienta a introdução das leitoas na vida reprodutiva recomenda que a primeira cobertura ou IA seja realizada a partir do segundo ou terceiro estro e quando a fêmea atingir pelo menos $120 \mathrm{~kg}$ de peso corporal, de forma a parir a primeira leitegada em torno dos $290 \mathrm{ou}$ mais dias de idade [9]. No entanto, resultados de outros trabalhos indicaram não haver benefícios econômicos em se realizar a primeira cobertura no segundo ou terceiro estro $[5,8,14,15]$.

O objetivo deste trabalho foi investigar a influência do estro em que foi realizada a primeira inseminação sobre a taxa de retorno ao estro, taxa de parto e tamanho da leitegada de fêmeas suínas em duas parições consecutivas.

\section{MATERIAIS E MÉTODOS}

O experimento foi conduzido em uma granja comercial destinada a multiplicação de matrizes localizada na região centro-oeste do estado do Rio Grande do Sul. Foram avaliadas 316 fêmeas híbridas da linhagem L291.

Os animais nasceram e foram criados na mesma granja onde ocorreu o experimento. Após completarem 160 dias de idade, as leitoas foram alocadas em baias contendo oito animais. No mesmo dia, teve início o manejo da estimulação do estro com o macho, que consistiu na introdução de machos adultos (>11 meses de idade) nas baias das leitoas por um período de 10 -15 minutos, duas vezes ao dia (8:00 h e as 17:00 h). As fêmeas que apresentam o reflexo de tolerância à monta pelo macho foram consideradas em estro. Estes animais foram alocados em novos lotes em baias coletivas para 8 animais de forma a se obter, em cada baia, um lote de leitoas ciclicamente síncronas.
A partir do $16^{\circ}$ dia após a manifestação do primeiro estro, as fêmeas foram controladas quanto à ciclicidade (o dia zero representado pelo primeiro dia do estro anterior). O controle do estro foi realizado de acordo com os procedimentos já anteriormente descritos. No momento do diagnóstico do segundo estro foram selecionados ao acaso os animais para formar três tratamentos, como segue:

Tratamento 1 (T1): fêmeas inseminadas no segundo estro pós-alojamento $(\mathrm{n}=103)$.

Tratamento 2 (T2): fêmeas inseminadas no terceiro estro pós-alojamento ( $\mathrm{n}=104)$.

Tratamento 3 (T3): fêmeas inseminadas no quarto estro pós-alojamento $(\mathrm{n}=109)$.

As inseminações artificiais ocorreram duas a três vezes por estro com uma dose inseminante de 90 mL contento $3 \times 10^{9}$ espermatozóides. A primeira IA ocorreu $12 \mathrm{~h}$ após o diagnóstico do estro e a segunda e terceira, em intervalos de 12 horas após a primeira IA. Foi utilizado um pool de dois ejaculados provenientes de machos com fertilidade comprovada.

A partir do $17^{\circ}$ pós-inseminação foi iniciado o controle dos retornos ao estro de acordo com os mesmos procedimentos utilizados para o diagnóstico do estro.

Após o parto e período de lactação médio de 19 dias, todas as fêmeas foram desmamadas e alojadas em celas individuais. Neste momento iniciou-se o controle quanto a apresentação do estro pós-desmame duas vezes ao dia (8:00 h e 17:00 h). Para este controle, um macho adulto foi colocado na frente dos lote de fêmas desmamadas. Um funcionário testou as fêmeas quanto a manifestação do reflexo de tolerância à monta pelo homem na presença de um macho. Na presença deste reflexo, as fêmeas foram consideradas em estro.

As variáveis resposta analisadas foram o porcentual de retornos ao estro ou taxa de parto, o porcentual de fêmeas que pariram ou taxa de parto e o número total de leitões nascidos por leitegada, ou seja, a soma do número de leitões vivos, mortos e mumificados.

As taxas de retorno ao estro e as taxas de parto foram comparadas pelo teste Exato de Fischer. As taxas de descarte no período entre o $1^{\mathrm{o}}$ parto e a $2^{\mathrm{a}}$ 
inseminação e o porcentual de fêmeas que completaram o segundo parto foram comparados pelo teste do Qui-quadrado. O número de leitões nascidos foi analisado por análise de variância e comparado pelo teste $t$ de Student. Para a análise dos dados utilizou-se o pacote estatístico JMP, Versão 5, SAS. ${ }^{2}$

\section{RESULTADOS}

Os animais do $\mathrm{T} 1(\mathrm{n}=103)$ apresentaram peso médio na inseminação artificial (PMIA) de 123 $\pm 10,7$ $\mathrm{kg}$ e idade média (IDM) de $185 \pm 18$ dias. O PMIA das fêmeas do T2 ( $\mathrm{n}=104)$ foi de $140 \pm 10,4 \mathrm{~kg}$, com IDM $207 \pm 20$ dias; fêmeas do T3 $(n=109)$ apresentaram $154 \pm 10,1 \mathrm{~kg}$ de PMIA e IDM de $227 \pm 19$ dias. A taxa média de retorno ao estro (TRE), taxa de parto e tamanho da leitegada nos grupos T1, T2 e T3 observados durante a primeiro gestação encontra-se na Tabela 1.

Tabela 1. Taxa de retorno ao estro, taxa de parto e número de leitões nascidos no primeiro parto das fêmeas nos três tratamentos.

\begin{tabular}{|c|c|c|c|c|c|}
\hline $\begin{array}{l}\text { Tratamen- } \\
\text { to }\end{array}$ & n & $\begin{array}{l}\text { Retorno } \\
*(n)\end{array}$ & $\begin{array}{l}\text { o Estro } \\
(\%)\end{array}$ & $\begin{array}{l}\text { Taxa de Parto* } \\
\text { (n) }(\%)\end{array}$ & $\begin{array}{l}\text { Leitões nascidos* } \\
\text { Média } \pm E P^{* *}\end{array}$ \\
\hline $\mathrm{T} 1$ & 108 & 4 & $3.70^{a}$ & $95.37^{b}$ & $10.44 \pm 0.26^{c}$ \\
\hline $\mathrm{T} 2$ & 106 & 3 & $2.83^{a}$ & $9690.57^{b}$ & $10.93 \pm 0.27^{c}$ \\
\hline T3 & 111 & 6 & $5.40^{\mathrm{a}}$ & $10190.99^{b}$ & $10.76 \pm 0.26^{c}$ \\
\hline
\end{tabular}

A taxa de descarte no período entre o $1^{\circ}$ parto e a próxima IA foi de $22,62 \%, 30,86 \%$ e $20,23 \%$ para as fêmeas do T1, T2 e T3, respectivamente $(\mathrm{P}>0,10)$.

A performance reprodutiva das fêmeas na segunda gestação está descrita na Tabela 2. Na segunda gestação, a taxa de retorno ao estro $(\mathrm{P}<0,05)$, taxa de parto $(\mathrm{P}<0,05)$, bem como o número médio de leitões nascidos $(\mathrm{P}<0,06)$ foi inferior nas fêmeas do tratamento T1 comparativamente às do T2 e T3. No entanto, não foi observada nenhuma diferença nestas variáveis entre os tratamentos T2 e T3 $(\mathrm{P}>0,1)$.

O porcentual de fêmeas que completaram dois partos e o número de leitões nascidos nestes partos em cada tratamento são apresentados na Tabela 3. O porcentual de fêmeas que completaram dois partos foi igual na comparação entre os tratamentos $(\mathrm{P}>0,1)$. Já o número de leitões nascidos nestes dois partos foi inferior no T1 comparativamente ao T2 e T3 $(\mathrm{P}<0,05)$.
Tabela 2. Taxa de retorno ao estro, taxa de parto e número de leitões nascidos no segundo parto das fêmeas nos três tratamentos.

\begin{tabular}{|c|c|c|c|c|c|c|}
\hline Tratamento & $n$ & $\begin{array}{l}\text { Retorno } \\
\text { (n) }\end{array}$ & $\begin{array}{c}\text { o Estro* } \\
(\%)\end{array}$ & $\begin{array}{c}\text { Taxa } \\
\text { (n) }\end{array}$ & $\begin{array}{l}\text { Parto* } \\
(\%)\end{array}$ & $\begin{array}{l}\text { Leitões nascidos } \\
\text { Média } \pm \text { EP }^{* * *}\end{array}$ \\
\hline $\mathrm{T} 1$ & 84 & 13 & $15,47^{b}$ & 68 & $80,95^{d}$ & $9.91 \pm 0.31^{f}$ \\
\hline T2 & 81 & 5 & $6,17^{\mathrm{a}}$ & 74 & $91.30^{\circ}$ & $10.51 \pm 0.30^{\circ}$ \\
\hline T3 & 84 & 5 & $5,95^{\mathrm{a}}$ & 77 & $91.67^{\circ}$ & $10.71 \pm 0.29^{e}$ \\
\hline
\end{tabular}

Tabela 3. Porcentual de fêmeas que completaram dois partos e o número total de leitões nascidos nos dois primeiros partos nos diferentes tratamentos.

\begin{tabular}{cllcc}
\hline Tratamento & $\mathbf{n}$ & \multicolumn{2}{c}{$\begin{array}{c}\text { Completaram } \\
\text { Dois Partos } \\
\mathbf{n}\end{array}$} & $\begin{array}{c}\text { Leitões Total } \\
\text { de Nascidos } \\
\text { Média } \pm E P^{* *}\end{array}$ \\
\hline T1 & 108 & 68 & $62,96^{\mathrm{a}}$ & $20,13 \pm 0,47^{\mathrm{c}}$ \\
T2 & 106 & 74 & $69,8^{\mathrm{a}}$ & $21,45 \pm 0,45^{\mathrm{b}}$ \\
T3 & 111 & 77 & $69,36^{\mathrm{a}}$ & $21,44 \pm 0,44^{\mathrm{b}}$ \\
\hline *- Letras diferentes na mesma coluna diferem para P£0,05. \\
** - Erro padrão
\end{tabular}

\section{DISCUSSÃO}

O tamanho da leitegada é determinado pela taxa de ovulação e pela taxa de sobrevivência embrionária. A taxa de ovulação aumenta nos primeiros estros pós-puberais, enquanto a taxa de sobrevivência embrionária parece declinar [2]. A taxa de ovulação e o tamanho da leitegada estão positivamente correlacionados até aproximadamente 18 ovulações. Uma vez atingido este valor, um aumento na taxa de ovulação resulta em pouco ou nenhum incremento no número de leitões nascidos [12].

No Brasil e utilizando genótipos atuais, foi observada uma taxa ovulatória média de 16,3 no segundo estro pós-puberal de leitoas com idade média de 186,3 dias [1]. Estes dados sugerem que os genótipos atuais apresentam, já no segundo estro pós-puberal, taxa ovulatória próxima ao ponto máximo descrito [12]. Desta forma, os resultados do presente estudo parecem indicar que as leitoas inseminadas no segundo (T1), terceiro (T2) e quarto estro (T3) apresentaram uma relação entre taxa ovulatória e sobrevivência embrionária semelhante, uma vez que o número de leitões nascidos no primeiro parto foi semelhante entre os tratamentos. Semelhantes tamanhos de leitegada também foram observados por outros autores [13] 
para leitoas inseminadas no segundo e terceiro estros com idades à inseminação de 181,5 e 204,9 dias, respectivamente.

No segundo parto, as fêmeas do T1 apresentaram maior taxa de retorno ao estro $(\mathrm{P}<0,05)$, menor taxa de parto $(\mathrm{P}<0,05)$ e menor número de leitões nascidos $(\mathrm{P}=0,06)$, quando comparadas com os grupos T2 e T3. Um menor tamanho da leitegada no segundo parto, também foram observados por outros autores $[7,10]$. Grandes perdas de estado corporal durante o período de lactação estão claramente associados com um declíneo na performance reprodutiva $[4,6,13]$.

No presente experimento, as fêmeas do T1 eram, em média, 20 dias mais jovens e, apresentavam menor tamanho corporal $(123 \pm 10,7 \mathrm{~kg})$ comparativamente às fêmeas do T2 $(140 \pm 10,4 \mathrm{~kg})$. Animais menores apresentam menor capacidade de ingestão. Esta menor capacidade de ingestão determinaria um maior catabolismo durante o período de lactação e conseqüentemente, esses animais desmamariam a primeira leitegada apresentando menor reserva corporal.

Segundo resultados descritos na literatura [3], porcas que perdem uma grande quantidade de proteína corporal (>9\%) durante o período de lactação apresentam menor número de folículos ovarianos ao desmame e, desta forma, são mais propensas a apresentarem menor taxa ovulatória e conseqüente diminuição do tamanho da leitegada. Além disso, uma menor sobrevivência embrionária pode também estar associada ao menor número de leitões nascidos e a maior taxa de retorno ao estro no $\mathrm{T} 1$ em relação aos demais tratamentos. São relatadas diferenças do estado metabólico no período de lactação que alteram significativamente as taxas de sobrevivência embrionária.
Existe, ainda, uma alta variabilidade individual no estado metabólico de primíparas durante a lactação, mesmo quando submetidas a condições semelhantes de manejo e nutrição [11]. Esta alta variabilidade individual pode sugerir que algumas fêmeas do T1 foram mais susceptíveis a perda do estado corporal durante o período de lactação e conseqüentemente apresentaram uma depressão da taxa ovulatória e da sobrevivência embrionária, o que poderia explicar a maior taxa de retorno ao estro e menor tamanho de leitegada no T1.

É importante salientar que neste trabalho, as leitoas foram selecionadas e iniciaram a vida reprodutiva na própria granja de origem, portanto, fatores relacionados com a aclimatação do animal e a adaptação à flora microbiana não foram fatores que interferiram nos resultados.

\section{CONCLUSÕES}

Conclui-se que leitoas inseminadas no segundo estro após o alojamento na granja apresentam performance reprodutiva semelhante na primeira gestação a leitoas inseminadas no terceiro ou quarto estro. No entanto, uma queda da performance reprodutiva dessa categoria de fêmeas pode ser esperada no segundo parto.

Agradecimentos. À FAPERGS e UNICRUZ pelo apoio financeiro e à Cooperativa Mista General Osório (COTRIBÁ-RS) pelo apoio financeiro e cedência dos animais e instalações.

\section{Agroceres PIC®.}

\section{Notas Informativas}

${ }^{2}$ SAS. Institute Inc., Cary, NC, 1989-2002.

\section{REFERÊNCIAS}

1 Afonso S.B. 1997. Efeito de Densidade e Lotação sobre os Índices Reprodutivos em Leitoas de Reposição. Porto Alegre, RS. Dissertação (Mestrado em Ciências Veterinárias) - Programa de Pós-graduação em Ciências Veterinárias, Universidade Federal do Rio Grande do Sul.

2 Archibong A.E., England D.C. \& Stormshak F. 1987. Factors contributing to early embryonic mortality in gilts bred at first estrus. Journal of Animal Science. 64: 474-478.

3 Clowes E.J., Aherne F.X., Schaefer A.L., Foxcroft G.R. \& Baracos V.E. 2003. Parturition body size and body protein loss during lactation influence performance during lactation and ovarian function at weaning in first-parity sows. Journal of Animal Science. 81: 1517-1528. 
4 Foxcroft G.R. 1996. Relationship between metabolism and reproduction. In: Proceedings of the 14th International Pig Veterinary Society Congress (Bologna, Italy). pp.6-9.

5 MacPherson R.M., Hovell F.D. \& Jones A.S. 1977. Performance of Sows First Mated at Puberty or Second or Third oestrus, and Carcass Assessment of Once-Bred Gilts. Animal Production. 24: 333-342.

6 Pettigrew J.E. 1998. Nutrition and prolificity. In: Proceedings of the 15th International Pig Veterinary Society Congress (Birmingham, UK). pp.319-323.

7 Reese D.E., Moser B.D., Peo E.R., Lewis A.J., Zimmerman D.R., Kinder J.E. \& Stroup W.W. 1982. Influence of energy intake during lactation on subsequent gestation, lactation, and postweaning performance of sows. Journal of Animal Science. 55: 590-598.

8 Rozeboom D.W., Pettigrew J.E., Moser R.L., Cornelius S.G. \& el Kandelgy S.M. 1996. Influence of gilt age and body composition at first breeding on sow reproductive performance and longevity. Journal of Animal Science. 74: 138-150.

9 Sancevero A.B. 1984. Aspectos genéticos do desempenho produtivo e reprodutivo de suínos em cruzamentos dialélicos. 142 f. Viçosa, MG. Tese (Doutorado em Ciências Veterinárias) - Programa em Ciências Veterinárias, Universidade Federal de Viçosa.

10 Whittemore C.T., Franklin M.F. \& Pearce B.S. 1980. Fat changes in breeding sows. Animal Production. 31: 183-192.

11 Willis H.J., Zak L.J. \& Foxcroft G.R. 2003. Duration of lactation, endocrine and metabolic state, and fertility of primiparous sows. Journal of Animal Science. 81: 2088-2102.

12 Wu M.C., Hentzel M.D. \& Dziuk P.J. 1987. Relationship between uterine length and number of fetuses and prenatal mortality in pigs. Journal of Animal Science. 65: 762-770.

13 Yang H.E.R. Easthan P., Philips P. \& Whittemore C.T. 1989. Reproductive performance, body weight and body condition of breeding sows with differing body weigh and body condition of breeding sows with differing body fatness at parturition, differing nutrition during lactation and differing litter size. Animal Production. 48: 181-201.

14 Young L.G., King L.G., Walton J.S., McMillian I. \& Klevorick M. 1990. Age, Weight, Backfat and Time of Mating Effects on Performance of Gilts. Canadian Journal of Animal Science. 70: 469-481.

15 Young L.G., King L.G., Walton J.S., McMillian I. \& Klevorick M. 1990. Reproductive Performance Over Four Parities of Gilts Stimulated to Early Estrus and Mated at First, Second or Third Observed Estrus. Canadian Journal of Animal Science.70: 483-492. 